\title{
A Retrospective Study of Efficacy and Toxicities of Rituximab in Combination with Chemotherapy for NHL patients in Bangladesh
}

\author{
*ME Hoque ${ }^{1}$, S Karim² ${ }^{2}$ S M N Hasan ${ }^{3}$, MMR Siddiqui ${ }^{4}$ \\ 1*Prof. Dr. Md Ehteshamul Hoque, Department of Oncology, Anwer Khan Modern \\ Medical College Hospital, Dhaka, Bangladesh \\ ${ }^{2}$ Dr. Shahnaz Karim, Department Of Transfusion Medicine \\ Dhaka Medical College Hospital, Dhaka, Bangladesh \\ ${ }^{3} S$ M Naimul Hasan, Department of Genetic Engineering and Biotechnology, University of Rajshahi \\ ${ }^{4}$ Dr. Md. Mahmudur Rahman Siddiqui, Assistant Professor of Medicine, Anwer Khan Modern \\ Medical College Hospital, Dhaka, Bangladesh
}

*Corresponding Author

\begin{abstract}
Bacground: In Bangladesh conventionally CHOP therapy is widely practiced for the treatment of NHL patient. But refractory/ relapse case is highly observed in CHOP therapy. Reports of different clinical trials showed that addition of Rituximab with CHOP therapy dramatically increase the progression free survival (PFS) and overall survival (OS) of patients.

Methodology: To analyze the response and toxicities of NHL patients treated with Rituximab in combination with other chemotherapy regimen admitted in Anwer Khan Modern Hospital, City Hospital and United Hospital in Dhaka, Bangladesh. Response and toxicities of patients admitted to three institutions between May 2003 to December 2006 were analyzed. A total of 30 patients were observed in this study. Patients received up to 6-8 cycles of Rituximab in combination with CHOP for every 3 weeks as an induction therapy. Maintenance therapy was given to 6 patients at 3 months interval for up to 2 years.

Result: After a median follow up period of 6 years patients were evaluated on the basis of response criteria. Out of the 30 patients 3 patients did not completed 6 cycle R-CHOP treatment regimen. $13 \%$ of the patients were refractory and died before completing therapy. With R-CHOP combination $47 \%$ of the patients experienced complete response. Partial response was observed in $20 \%$ of the patients. In this study $6 \%$ of the patients did not get response from the therapy. Haematological toxicity included grade 3-4 neutropenia 13\%, anaemic $4 \%$, febrile neutropenia $17 \%$ and thrombocytopenia $11 \%$. Non-haematological toxicity included grade $2-3$ nausea or vomiting $18 \%$, grade 3 fatigue $12 \%$.

Conclusion: Addition of Rituximab as an immunotherapy option with chemotherapy regimen provides excellent response to Bangladeshi patients diagnosed for Diffused Large B Cell Lymphoma (DLBCL). Further study needs to be done to evaluate efficacy and toxicity profiles of patients treated with other chemotherapy regimen who were not eligible for CHOP regimen.
\end{abstract}

Key Words: Rituximab, non-Hodgkin's lymphoma (NHL), Diffused Large B Cell Lymphoma, R-CHOP

\section{Introduction}

Diffuse large B-cell lymphoma (DLBCL) is the most common high-grade lymphoma and the most common subtype of non-Hodgkin's lymphoma $(\mathrm{NHL})^{1,2}$. In recent years, the incidence of NHL has been increasing not only in western countries but also in the Asia-Pacific region, including Bangladesh. According to the recent clinic pathological investigation of malignant lymphoma in Bangladesh, B-cell NHL accounted for $88 \%$ of the total NHL cases, and its major subtype was DLBCL15. Aggressive NHL, represented by DLBCL, is classified as a curable disease.
However, the cure rate with standard chemotherapy is as low as $30-40 \%{ }^{4,5,1,7}$.

Rituximab, a chimeric anti-CD20 monoclonal antibody, was originally studied in patients with relapsed and refractory follicular and low-grade NHL and clearly was shown to prolong progressionfree survival ${ }^{8,9}$. The mechanism of action is based on antibody-dependent cellular cytotoxicity, complement induced cytotoxicity, and direct induction of apoptosis ${ }^{10,11,12,13,14}$. Because CD20 is a pan- $\beta$-cell antigen ${ }^{15}$, rituximab has potential application in many $\beta$-cell NHL and continues to be assessed, both alone and in combination with 
chemotherapy, for the management of patients with indolent and aggressive NHL. The feasibility and safety of combining cyclophosphamide, doxorubicin, vincristine, and prednisone (CHOP) with rituximab was demonstrated in a phase II trial in aggressive lymphoma, which reported a higher than expected complete remission rate of $61 \% 16$. The Group d'Etudes des Lymphomes de l'Adulte (GELA) recently reported that using the combination of rituximab plus CHOP leads to the significant improvement of the outcome of elderly patients with DLBCL, with significant survival benefit maintained during a 10-year follow-up ${ }^{17,6}$.

An Intergroup study under the lead of the Eastern Cooperative Oncology Group (ECOG) (E4494) was able to confirm these results. The German highgrade study group also reported that the addition of rituximab to six cycles of a $\mathrm{CHOP}$-like regimen significantly improves time to treatment failure, CR rates, and overall survival (OS) in young patients (18-60 years) with low-risk DLBCL ${ }^{11,18}$. After the addition of rituximab to CHOP chemotherapy, the outcome has dramatically improved compared with the historical control for DLBCL patients of all ages in British Columbia ${ }^{13,19}$. These positive results suggested that rituximab plus CHOP (R-CHOP) could be a new standard treatment strategy for patients with DLBCL. However, these encouraging reports have been limited in western countries. Through a Japanese trial, $375 \mathrm{mg} / \mathrm{m}^{2}$ was suggested as the proper dose for oriental patients ${ }^{18,22}$. We conducted a retrospective analysis to evaluate the impact of this combination therapy with diffuse large $\beta$-cell lymphoma (DLBCL) in Bangladesh. A total of 30 patients with DLBCL were evaluated in this study.

\section{Materials and Methods}

\section{Patient's selection and evaluation}

Patients were treated at the Oncology Department of Anwer Khan Modern Medical College \& Hospital, Dhaka, United Hospital Ltd, Dhaka and City Hospital Ltd, Dhaka. The patient population in this study consisted of newly diagnosed patients of at least 18 years of age with histologically documented aggressive lymphoma (IWF types D to H). Patients with mantle-cell, lymphoblastic, or Burkitt's lymphoma were excluded, because CHOP is not considered routine treatment in these patients. The stage of each patient's disease was assigned according to the Ann Arbor classification. Tumors were required to be CD20-positive. Patients were expected to have a survival of 6 months or more and a pre study performance status of 0,1 , or 2 according to the World Health Organization scale. The following exclusion criteria were applied: history of transformation from a low-grade lymphoma or history of a T-cell lymphoma, presence of CNS lymphoma, human immunodeficiency virus, human T-cell leukemia virus 1 or 2 positivity, prior anticancer therapy, significant organ function impairment as measured by serum creatinine level greater than $2.0 \mathrm{mg} / \mathrm{dL}$, a total bilirubin level greater than $2.0 \mathrm{mg} / \mathrm{dL}$, or an AST or alkaline phosphatase level more than two times normal, hemoglobin concentration less than 9 $\mathrm{g} / \mathrm{dL}$ or absolute neutrophil count less than $1.5 \times 103 / \mu \mathrm{L}$, previous or concomitant malignancy other than basal cell or squamous cell carcinoma of the skin, and carcinoma-in-situ of the cervix. Patients with New York Heart Association class III or IV heart disease or myocardial infarction within the past 6 months were disqualified from entering onto the study. Pregnant or lactating women and patients of reproductive potential, unless using accepted birth control methods, were not allowed to enroll.

\section{Treatment Design}

This study consisted of a single treatment group. Patients were to receive a total of six to eight intravenous infusions of Rituximab $375 \mathrm{mg} / \mathrm{m}^{2}$ and six cycles of CHOP given every 21 days. Rituximab infusions were administered on day 1 before the CHOP cycle. Each CHOP cycle consisted of cyclophosphamide $750 \mathrm{mg} / \mathrm{m}^{2}$, doxorubicin $50 \mathrm{mg} / \mathrm{m}^{2}$, and vincristine $1.4 \mathrm{mg} / \mathrm{m}^{2}$ (maximum dose, $2.0 \mathrm{mg} / \mathrm{dose}$ ) given intravenously on day 3 , and oral prednisone $100 \mathrm{mg}$ on days 3 through 7 .

Oral premedication with $650 \mathrm{mg}$ of acetaminophen and 50 to $100 \mathrm{mg}$ of diphenhydramine hydrochloride could be administered 30 to 60 minutes before each monoclonal antibody infusion. If toxicity occurred during the monoclonal antibody infusion, the infusion was to be slowed or temporarily discontinued and the patients were to be medicated as necessary with diphenhydramine (for rash, mucosal congestion, or other infusion-related reactions), and other medications were administered as needed. Once the adverse events abated, the antibody infusion could be resumed at $50 \%$ of the previous rate and then escalated as tolerated. 


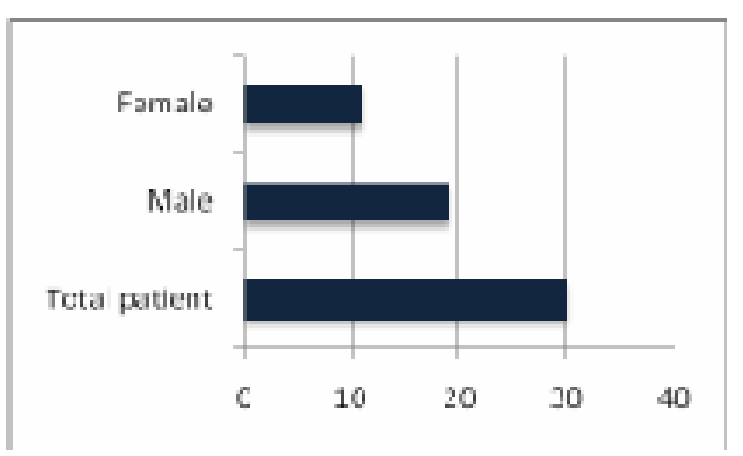

Figure-1: Patient characteristics in the study

CHOP was to be administered according to standard preparation and procedures for each institution. Cyclophosphamide dose modification for hematologic toxicities was to be carried out according to an algorithm provided in the protocol. If grade 3 neurotoxicity occurred at any time during the treatment period, vincristine could be discontinued at the investigator's discretion. Hematopoietic growth factors (granulocyte or granulocyte-macrophage colony-stimulating factor) were administered per institutional guidelines. A patient whose treatment was interrupted for more than 3 weeks for either hematologic or nonhematologic toxicity was to be removed from the study.

\section{Toxicity and Response Assessments}

Hematological and non-hematological toxicity evaluation was conducted on day 1 of each treatment cycle and included neurological examination and laboratory assessment with complete blood cell count and serum chemistry tests. Thoracic, abdominal and pelvic CT scans and bone marrow biopsy (in patients with bone marrow involvement at initial diagnosis) were conducted to assess response after both induction therapy and completion of consolidation therapy. After four treatment cycle (induction therapy), patient achieving a complete response (CR), unconfirmed complete response $(\mathrm{CRu})$ or partial response $(\mathrm{PR})$ were eligible for to two further cycles of R-CHOP.

\section{Statistical Analysis}

Patient characteristics and therapeutic outcomes were compared in this study. This was an intentionto-treat analysis, including all patients treated with curative intent who received at least three cycles of RCHOP or CHOP chemotherapy. Clinical prognostic factors were also compared between two groups including conventional international prognostic index (IPI) score, using the independentsamples $\mathrm{t}$ test for continuous variables and the ?2 test for categorical variables. Event free survival (EFS) was calculated as the time from date of diagnosis to documented disease progression, relapse, or other events including documented failure of the treatment not associated with disease progression. OS was calculated as the time from date of diagnosis to date of death. EFS and OS were assessed using the Kaplan-Meier method and compared between groups using the log-rank test. A multivariate analysis was performed using the Cox proportional hazards model to assess the independent effect of the addition of rituximab onEFS and OS after controlling for relevant clinical prognostic factors. We also conducted analysis divided into each subgroup according to the IPI and other clinical prognostic factors. Data were analyzed using the Statistical Software Package for the Social Sciences (SPSS version 11.0 for Windows; SPSS, Chicago, IL, USA).

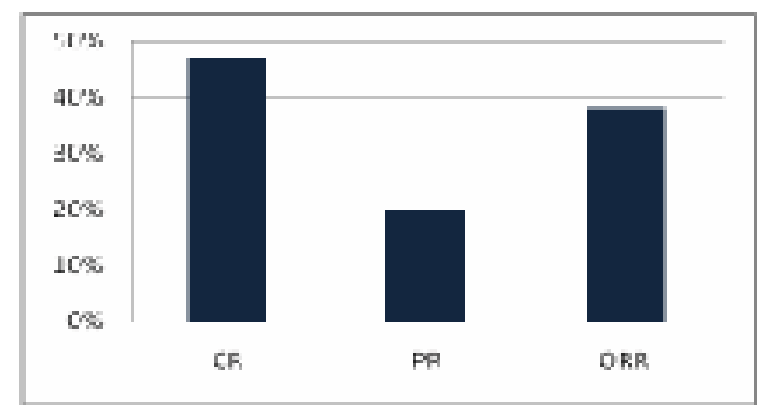

Figure-2: Outcome of the NHL patients of Bangladeshi population treated with Rituximab

\section{Results}

Response and toxicities of patients admitted to three institutions between May 2003, and December 2006 were analyzed. The median age of all 30 patients was 57 years old (range 35-72). Among them male patients were 19 and female patients were 11 . Pretreatment patient characteristics are summarized in Figure 1. The male to female ratio was 1:2. There were no statistically significant differences between the two groups, except for age. Other patients' demographics such as, performance status (PS), lactate dehydrogenase (LDH), stage, and IPI showed no definite difference between the two groups. Many of the patients (83\%) had good PS of $0-1$ score in ECOG scale. Thirty-five percent of the patients had stage III or IV. Higher LDH level was found in $41 \%$ of the patients. The vast majority $(74 \%)$ had low or low-intermediate risk according to the IPI. 


\section{Patient Disposition}

Three patient numbers did not receive all six cycles of rituximab doses. These three patient numbers represent a single individual who was re-treated within the study and received a single infusion under each patient number. The other 27 patient numbers received all six scheduled doses.

\section{Response to Treatment}

In the 27 assessable patients, $47 \%$ of the patients experienced CRs and $20 \%$ PRs, as listed in Table. The ORR was $38 \%$ in the 30 intent-to-treat patients, with thirteen CRs and 6 PRs. Treatment response is summarized in Figure 2. The median time to response was 49 days. The median DR and TTP in responders have not been reached; Kaplan-Meier median estimates are 17.8 months for DR (range, 5.41 to 26.6 months) and 16.3 months for TTP (range, 3.71 to 25.1 months). Responses are ongoing in fifteen of the 24 responding patients. Of the 2 responders who relapsed, one patient experienced PD because of new lesions and other relapsed at sites of old lesions. Tumor size was reduced substantially in most patients. The average decrease in lesion size was $86 \%$ in patients achieving a PR and $32 \%$ in patients with SD. Tumor size was reduced by at least $20 \%$ in $73 \%$ of patients (19 of 27). Thirty percent of patients (9 of 30) reported B symptoms or other disease-related signs and symptoms at baseline, and symptoms resolved completely or were relieved transiently in $16(86 \%)$ of 25 patients. Two patients did not get response from the treatment protocol.

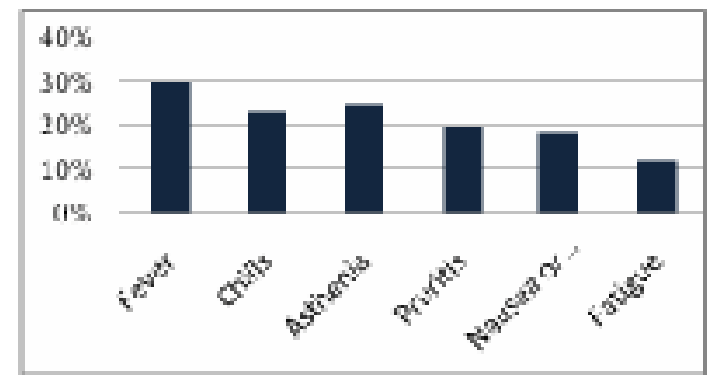

Figure-3: Non hematologic side effects in the treated population

\section{Clinical Adverse Events}

Most clinical AEs (92\% of events) were mild to moderate (grade 1 or 2 ) and self limiting. The majority of patients experienced AEs during the first infusion, and both incidence and frequency declined with subsequent infusions. Approximately half of all events were considered to be related (probably or possibly related, or of unknown relationship) to the study treatment. Of grade 3 or 4 events, $69 \%$ were considered unrelated to the treatment. The most common related events observed during the treatment period ( 22 days of treatment plus 30 days of follow-up) included transient fever (30\% of patients), chills (23\%), asthenia (25\%), and pruritus (20\%), grade $2-3$ nausea or vomiting $18 \%$, grade 3 fatigue $12 \%$. Infusion-related events (related events reported on an infusion day) included episodes of fever, chills, pain, and throat irritation that were grade 1 or 2 , usually continued for less than 1 week, and resolved without treatment or without interrupting the infusion. Infections were generally mild to moderate and only two patients with infection required hospitalization. Significant myelosuppression was not observed; hematologic toxicity was generally mild and reversible.

Nine patients developed grade 3 neutropenia approximately 1 month after the fourth infusion on this protocol. The neutropenia resolved with granulocyte colony-stimulating factor (G-CSF) therapy. These patients had also developed similar neutropenia approximately 1 month after completion of his initial rituximab therapy. A marrow sample taken during the nadir showed only regenerating myeloid elements with no malignant involvement, and with a thorough work up, no other infectious or toxic cause for his marrow suppression was found. Again, the neutropenia responded to G-CSF treatment.

\section{Hematology and Chemistry Laboratory Effects}

Haematological toxicity included grade 3-4 neutropenia (13\%), anaemic (4\%), febrile neutropenia (17\%) and thrombocytopenia (11\%). During the treatment period, hemoglobin levels declined to less than $8 \mathrm{~g} / \mathrm{dL}$ and absolute neutrophil count declined to less than $1,000 / \mathrm{mL}$ in one patient each; WBC was less than $2000 / \mathrm{mL}$ in two patients. During the 1-year follow up, absolute neutrophil count declined to less than $1,000 / \mathrm{mL}$ in two patients. Levels of these hematologic parameters recovered within 2 weeks in three patients and by 4 months in one patient; levels in one patient had not recovered by database closure. No hepatic or renal toxicity was noted. No patient developed HACA. Mean serum immunoglobulin levels did not decrease below the normal range. 


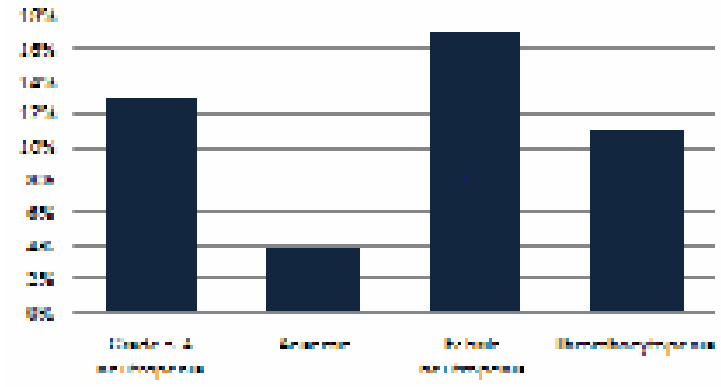

Figure-4: Hematologic side effects in the treated population

\section{Discussion}

The treatment of aggressive non-Hodgkin's lymphoma has been one of the major success stories in cancer therapy. However, despite the development of newer chemotherapeutic agents and more intensive chemotherapy regimens, no improvement in outcome has been demonstrated during the last three decades. ${ }^{5}$

Advances in molecular biology have allowed the development of newer targeted therapies. In 1997, rituximab became the first monoclonal antibody approved by the US Food and Drug Administration for cancer therapy. ${ }^{8}$ Rituximab targets the CD20 cell surface protein, present on mature $\beta$ cells and most B-cell malignancies. Its effects are likely multifactorial and include complement-dependent cell lysis, cell-mediated cytotoxicity, and induction of apoptosis $19,12,20$. Preclinical models suggested a synergistic effect when rituximab was combined with chemotherapy. ${ }^{20,21}$ and early clinical trials demonstrated minimal increase in toxicity. ${ }^{22,23}$

The GELA trial demonstrated that the addition of rituximab to $\mathrm{CHOP}$ chemotherapy improved outcome in elderly patients with advanced-stage DLBCL. ${ }^{24}$ The current study is a population-based analysis designed to evaluate the impact of the introduction of this combination therapy across the general population of patients with advanced stage DLBCL. Our results confirm a dramatic improvement in outcome after the adoption of a treatment policy combining CHOP and rituximab for all patients with advanced stage DLBCL, regardless of age. Patients treated in the postrituximab era had an $18 \%$ absolute improvement in 2-year PFS and a $25 \%$ improvement in 2-year OS compared with those treated in the prerituximab era. The addition of rituximab resulted in an approximately 50\% decrease in the risk of dying within the first 2 years after diagnosis.
Although our series was a retrospective analysis, the present study showed a few unique points compared with other series. All patients in our series had DLBCL and were treated with CHOP or R-CHOP. As shown in patients' characteristics, there is no difference between the groups except for age. The better outcome in this study was more prominent in high-risk patients according to the IPI. However this statistical significance was masked in low-risk patients. These results suggest that rituximab may contribute to the improvement of the treatment for high-risk patients. Recently, GELA group reported that rituximab addition could overcome bcl-2associated resistance to chemotherapy ${ }^{26}$. Although our study cannot confirm this correlation due to inadequate data of bcl-2, rituximab may contribute to the benefit for the high-risk group according to the IPI in regards to bcl-2 expression is frequently associated with poor outcome. Our population also had more patients of low risk in IPI than GELA trial. This different distribution of risk group may contribute to better outcome of high-risk group to the RCHOP.

This finding may also provide further evidence that front-line high-dose chemotherapy followed by stem cell transplantation for high-risk patients could be replaced by R-CHOP. As previously shown, toxicity for this regimen was mild and feasibility was good. The GELA LNH 98.5 and MInT trials showed that the addition of rituximab could improve the outcome in both young and elderly patients ${ }^{27,11}$. The present study confirmed this benefit of rituximab added to CHOP regardless of age for the first time in Bangladeshi patients. Through subgroup analysis, it was confirmed that patients with localized stage as well as advanced stage could get benefit from rituximab. In spite of the short follow-up, significant improvement in EFS was observed as well in the group of patients with localized disease that received involved field radiotherapy in addition to $\mathrm{CHOP}$ with rituximab. This result suggests that a short course of CHOP plus radiation therapy (RT) for patients with localized disease may be replaced with RCHOP plus RT for patients with good prognosis. A recent trial reported that R-CHOP had benefit for patients with early-stage gastric DLBCL ${ }^{25}$. As shown by these results, the addition of rituximab may extend the indication beyond classic DLBCL. 
In this trial, independent of pathologic grade and treatment intent, rituximab showed remarkable effect for the treatment of $\beta$-cell $\mathrm{NHL}^{27,28}$. Our population included a variety of DLBCL, including extranodal presentation. The biologic characteristics of these subtypes are yet to be defined and could not be performed in this retrospective analysis. This limitation will be overcome by further investigation of the biologic background through newer methods including tissue microarrays.

\section{Conclusion}

In conclusion, the addition of rituximab to standard CHOP chemotherapy in patients with newly diagnosed DLBCL showed markedly improved outcome in Bangladeshi patients. This better outcome was prominent in high-risk patients according to the IPI. RCHOP plus RT is expected to be the new therapeutic option for patients with localized DLBCL. Independent of IPI score, rituximab addition had a very positive impact on outcomes in the treatment of DLBCL.

\section{Conflict of interest: none}

\section{References}

1. Fisher RI, Miller TP, and O'Connor OA et al. Diffuse aggressive lymphoma. Hematology, 2004; 221-236.

2. Jaffe ES, Harris NL, Stein H et al. Tumours of haematopoietic and lymphoid tissue in World Health Organization Classification of Tumors. IARC Press, Lyon, 2001; pp 171174

3. Hennessy BT, Hanrahan EO, Daly PA et al. Non-Hodgkin's lymphoma: an update. Lancet Oncol 2004; 5:341-353.

4. A Akhter, MR Rahman, N Majid, S Shermin, MS Saleheen, RC Rajib, SMA Ullah, $\mathrm{N}$ Haque, AK Akond et al. Histological subtypes of Non-Hodgkin's Lymphoma in different age and sex groups, Bangladesh Medical Journal 2012; Vol. 41 No. 1:32-36

5. Cheson BD What is new in lymphoma? Cancer J Clin, 2005; $54: 260-272$.

6. Fisher RI, Gaynor ER, Dahlberg S et al. Comparison of a standard regimen (CHOP) with three intensive chemotherpay regimens for advanced non-Hodgkin's lymphoma. N Engl J Med 1993; 328:1002-1006.

7. Gordon LI, Harrington D, Andersen J et al. Comparison of a second-generation combination chemotherapeutic regimen (mBACOD) with a standard regimen (CHOP) for advanced diffuse non-Hodgkin's lymphoma. N Engl J Med 1992; 327:1342 1349 .
8. Foran JM, Rochatiner AZS, Conningham et al. European phase II study of rituximab (chimeric anti-CD20 monoclonal antibody) for patients with newly diagnosed mantle-cell lymphoma, immunocytoma, and small B-cell lymphocytic lymphoma. J Clin Oncol 2000; 18:317-324.

9. McLaughlin P, Grillo-Lopez AJ, Link BK et al. Rituximab chimeric anti-CD20 monoclonal antibody therapy for relapsed indolent lymphoma: half of patients respond to a four-dose treatment program. J Clin Oncol 1998; 16:28252833.

10. Demidem A, Hanna N, Hariharan $\mathrm{H}$ et al. Chimeric antiCD20 antibody (IDEC C2B8) is apoptotic and sensitizes drugresistant human B cell lymphomas to the cytotoxic effect of CDDP, VP-16 and toxins. FASEB J 1995; 9:A206

11. Pfreundschuh MG, Trumper L, Ma D et al. Randomized intergroup trial of first line treatment for patients $<60$ years with diffuse large B-cell non-Hodgkin's lymphoma (DLBCL) with a CHOP-like regimen with or without the anti-CD20 antibody rituximab - early stopping after the first interim analysis. J Clin Oncol 2004; 22:14S (abstract no. 6500).

12. Reff ME, Carner K, Chambers KS, Chinn PC et al. Depletion of $\mathrm{B}$ cells in vivo by a chimeric mouse human monoclonal antibody to CD20. Blood 1994; 83:435-445.

13. Sehn LH, Donaldson J, Chhanabhai $\mathrm{M}$ et al. Introduction of combined CHOP plus rituximab therapy dramatically improved outcome of diffuse large B-cell lymphoma in British Columbia. J Clin Oncol 2005; 23:5027-5033.

14. Shan D, Ledbetter JA, Press OW et al. Apoptosis of malignant human B cells by ligation of CD20 with monoclonal antibodies. Blood 1998; 91:1644-1652.

15. Chang KL, Arber DA, Weiss LM et al. CD20: a review. Appl Immunohistochem 1996; 4:1-15.

16. Vose JM, Link BK, Grossbard ML et al. Phase II study of rituximab in combination with CHOP chemotherapy in patients with previously untreated, aggressive non-Hodgkin's lymphoma. J Clin Oncol 2001; 19:389-397.

17. Coiffier B, Thieblemont C, Van Den Neste E, Lepeu G, Plantier I, Castaigne S, Lefort S, Marit G, Macro M, Sebban C, Belhadj K, Bordessoule D, Fermé C, Tilly H et al. Long-term outcome of patients in the LNH-98.5 trial, the first randomized study comparing rituximab-CHOP to standard CHOP chemotherapy in DLBCL patients: a study by the Groupe d'Etudes des Lymphomes de l'Adulte. Blood. 2010 Sep 23; 116(12):2040-5.

18. Tobinai $\mathrm{K}$, Igarashi $\mathrm{T}$, Itoh $\mathrm{K}$ et al. Japanese multicenter phase II and pharmacokinetic study of rituximab in relapsed or refractory patients with aggressive B-cell lymphoma. Ann Oncol 2004; 15:821-830. 
19. Anderson DR, Grillo-Lopez A, Varns C, et al. Targeted anticancer therapy using rituximab, a chimaeric anti-CD20 antibody (IDEC-C2B8) in the treatment of non-Hodgkin's Bcell lymphoma. Biochem Soc Trans 1997; 25:705-708.

20. Demidem A, Lam T, Alas S, et al. Chimeric anti-CD20 (IDEC-C2B8) monoclonal antibody sensitizes a B cell lymphoma cell line to cell killing by cytotoxic drugs. Cancer Biother Radiopharm 1997; 12:177-186.

21. Chow KU, Sommerlad WD, Boehrer S, et al. Anti-CD20 antibody (IDEC-C2B8, rituximab) enhances efficacy of cytotoxic drugs on neoplastic lymphocytes in vitro: Role of cytokines, complement, and caspases. Haematologica 2002; 87: 33-43.

22. Vose JM, Link BK, Grossbard ML, et al. Phase II study of rituximab in combination with CHOP chemotherapy in patients with previously untreated, aggressive non-Hodgkin's lymphoma. J Clin Oncol 2001; 19:389-397.

23. Czuczman MS, Grillo-Lopez AJ, White CA, et al. Treatment of patients with low-grade B-cell lymphoma with the combination of chimeric anti-CD20 monoclonal antibody and CHOP chemotherapy. J Clin Oncol 17:268-276, 1999.
24. Coiffier B, Lepage E, Briere J, et al. CHOP chemotherapy plus rituximab compared with $\mathrm{CHOP}$ alone in elderly patients with diffuse large- B-cell lymphoma. N Engl J Med 2002; 346:235-242.

25. Wohrer S, Puspok A, Drach J et al. Rituximab, cyclophosphamide, doxorubicin, vincristine and prednisone (R-CHOP) for treatment of early-stage gastric diffuse large B cell lymphoma. Ann Oncol 2004; 15:1086-1090.

26. Mounier N, Briere J, Gisselbrecht $\mathrm{C}$ et al. Rituximab plus CHOP (R-CHOP) overcomes bcl-2-associated resistance to chemotherapy in elderly patients with diffuse large B-cell lymphoma (DLBCL). Blood 2003; 101:4279-4284

27. Feugier P, Van Hoof A, Sebban C et al. Long-term results of the R-CHOP study in the treatment of elderly patients with diffuse large B-cell lymphoma: a study by the Groupe d'Etudes des Lymphomes de l'Adulte. J Clin Oncol 2005; 23:4117- 4126.

28. Coiffier B Current strategies for the treatment of diffuse large $\beta$ cell lymphoma. Curr Opin Hematol 2005; 12:259-265. 